\title{
HIGH-ENERGY NUCLEAR PHYSICS
}

\author{
PHYSICAL SQCIETY'S SUMMER MEETING
}

T HERE are now three machines in Great Britain which can produce $\pi$-mesons in useful numbers. These are the $1,000-\mathrm{MeV}$. proton synchrotron at Birmingham, the $400-\mathrm{MeV}$. synchro-cyclotron at Liverpool and the $300-\mathrm{MeV}$. electron synchrotron at Glasgow. All three accelerators have been in successful operation for some time, and the first experimental results are beginning to appear. It was therefore timely for the Physical Socitty to devote the whole of its provincial summer meeting, held during July 5-6, to high-energy nuclear physics. The choice of Liverpool as a meeting place was particularly appropriate in view of the striking success recently achieved there in the extraction of the beam from the synchro-cyclotron. Members of the Society and visitors had an opportunity during the meeting of visiting the Nuclear Research Laboratory of the University, where, in addition to the 400-MeV. synchro-cyclotron, a 37-in. eyclotron and a $\mathrm{I}-\mathrm{MeV}$. high-tension accelerator are also in operation. The average attendance at the meetings was about 150 , and some seventy members joined in the conference dinner held in the University Staff House on July 5 .

The scientific programme was preceded by a short business meeting, during which the president of the Physical Society (Prof. H. S. W. Massey) expressed his pleasure at the opportunity offered to the Society of visiting Liverpool. In reply to this, Prof. H. W. B. Skinner welcomed the Society, and in particular a number of overseas visitors, to what was possibly the first conference on work with high-energy machines outside the United States. The subsequent papers and discussions left little doubt that high energy physics is now a flourishing subject in Great Britain. It was particularly interesting to note that the high-energy accelerators are being used by groups of workers from many laboratories, and that collaboration of this sort is likely to increase. In the present report, only the names of those actually presenting the papers are given, although those of their co-workers were in general scrupulously announced at the meeting.

The papers presented on the first day were all concerned with the interaction of high-energy neutrons and protons with matter. Several papers described work carried out at Harwell, where the $150-\mathrm{MeV}$. synchro-cyclotron, although not useful for $\pi$-meson production, was the first high-energy accelerator to start working in Britain, and has boen used for a large number of precise experiments in the high-energy field. T. C. Randle (Atomic Energy Research Establishment, Harwell) described a new determination of the angular distribution in $(n, p)$ scattering at $130 \mathrm{MeV}$., which clearly shows a marked asymmetry between backward and forward scattering. T. C. Griffith (University College, London) later described another determination of the differential cross-section for $(n, p)$ scattering using the neutron beam of the Harwell cyclotron with photographicemulsion recording of the recoil protons. His results cover the angular range $57 \frac{1}{2}-82 \frac{1}{2}{ }^{\circ}$ in the laboratory system and agree with those of other workers within the limits of error. Some observations of the charged particles emitted by carbon under neutron bombard. ment were obtained in the course of this work. P. Hillman (Harwell) reported on the observation of polarization in the scattering of $100-\mathrm{MeV}$. neutrons by free protons. This is an extremely diffeult experiment, but good evidence for a real effect was presented, and the chairman (Prof. H. S. W. Massey) emphasized its importance for a proper analysis of nucleon-scattering work. Another interesting and informative experiment of a similar nature using the $100-\mathrm{MeV}$. neutrons from the Harwell machine was described by R. Wilson (Oxford). He and his colleagues have been able to observe the Coulomb scattering of neutrons, due to the motion of the neutron magnetic moment in the electric field of the nucleus. This scattering was predicted by Schwinger and is observable only at forward angles of less than $1^{\circ}$. In this region, interference between the Coulomb and nuclear seattering gives a polarization effect and enables the polarization of the initial neutron beam to be demonstrated. J. M. Dickson (Harwell) described polarization effects observed at angles between $3^{\circ}$ and $20^{\circ}$ in the scattering of polarized protons by carbon, aluminium, copper and silver. 'The effect of the interference between Coulomb and nuclear scattering in the asymmetry was very clearly shown. Both the $(p, p)$ and the $(n, p)$ experiments were remarkable for the angular precision achieved. Later in the meeting, A. Roberts (Rochester, U.S.A., and U.S. Office of Naval Research, London) reported on earlier $(n, p)$ polarization experiments at Rochester which are in agreement with the Harwell results, and cover a wider energy range. He also described $(p, p)$ polarization work and showed how the polarization produced in the scattering of protons by carbon is zero near the first diffraction minimum.

L. Riddiford (Birmingham) described the results of a study of the $(p, p)$ interaction at about $650 \mathrm{MeV}$. using protons accelerated in the Birmingham synchrotron. At this energy the differential cross-section for elastic scattering may be influenced by the possibility of meson production, and a forward peak might be expected, in contrast with the isotropic distribution obtained at lower energies. The results for 131 events, which were obtained with a diffusion cloud chamber filled with hydrogen to a pressure of 25 atmospheres and operating in a magnetic field of $13 \mathrm{k}$.gauss show this feature. Of the inelastic events in hydrogen, forty-five examples of $\pi^{+}$-meson production and nine examples of $\pi^{0}$-meson production were identified. Angular correlation measurements made for the cases of $\pi^{+}$-meson production were in agreement with the hypothesis of an excited state of the nucleon-meson system corresponding to the $T=3 / 2$ resonance observed in meson-nucleon scattering. W. O. Lock (Birmingham) reported on a similar study using $950-\mathrm{MeV}$. protons from the Birmingham machine. Collisions with hydrogen were obtained and recorded in nuclear emulsions, and the events were studied independently at Birmingham, Belfast and Glasgow. In this work a marked deviation from isotropy was also seen at forward angles, in. cluding a dip near $0^{\circ}$, which may still be due to poor statistics. The experiment gives a cross-section for $(p, p)$ elastic scattering at $950 \mathrm{MeV}$. of $15 \cdot \overline{0} \pm 3 \mathrm{mb}$.; no evidence for appreciable polarization of the beam 
scattered out of the synchrotron at $4^{\circ}$ was found. In the sarne exposures 'quasi-elastic' collisions of the incident proton with bound protons in the heavier nuclei of the emulsion are recorded, and identification and analysis of these events was described by W. M. Gibson (Belfast).

Some discussion took place on the effort spent in analysing experiments made with photographic plates and diffusion cloud chambers. The great experimental advantages conferred by the availability of a continuous, intense and homogeneous external beam were clearly brought out in a paper by J. R. Holt (Liverpool) on the elastic scattering of protons by hydrogen at $380 \mathrm{MeV}$. The differential cross-section at $90^{\circ}$ was found by coincidence counting using the carbon-poly thene difference method; and a result of 3.63 millibarns, with an accuracy of about 1 per cent, was obtained. The excellent conditions available at Liverpool for precision high-energy experiments in the $400 \mathrm{MeV}$. region were well shown in this work. Another example of the type of work now possible at Liverpool was given by $\mathbf{A}$. Ashmore, who presented the results of measurements of neutron total cross-sections for a number of elements using the transmission method with a scintillation telescope.

Towards the end of the first day, Prof. Massey reviewed progress in high-energy scattering and polarization phenomena, referring in particular to the very precise work which has now been reported from the $660-\mathrm{MeV}$. Moscow synchro-cyclotron. $\mathrm{He}$ stressed the fact that advances in experimental technique have been extremely rapid, and that it is now possible to envisage triple- and quadruple-scattering experiments which could determine uniquely the unknown parameters in the general nucleon-nucleon scattering matrix.

The second day of the meeting was devoted to miscellaneous topics. The Glasgow electron synchrotron was described by W. Macfarlane; it now produces $4 \times 10^{10}$ equivalent quanta per min. for a pulse-rate of 5 per sec. and an energy of $340 \mathrm{MeV}$., which appears to be better than the performance of comparable American machines. The beam has been collimated to $\frac{1}{4}$ in. diameter at $125 \mathrm{~cm}$. from the synchrotron. Arrangements for monitoring the beam with a pair spectrometer were described and some excellent expansion-chamber pictures of photoninduced stars in air were shown. The Glasgow synchrotron will be used in part for a study of the photoproduction of $\pi$-mesons, and E. H. Bellamy reported preliminary observations on the ratio of $\pi^{+}$. to $\pi^{-}$-mesons produced in calcium; nuclear emulsion detectors' were used, and the ratio was found for three energy ranges. R. G. Moorhouse (Glasgow) reported on new calculations on the photoproduction of mesons, referring in particular to the effect of $E 2$ transitions on the angular distribution of mesons.

Several papers were devoted to the properties of $\pi$-mesons. S. W. Barnes (Rochester, U.S.A.) described measurements of the absolute differential crosssection for the elastic scattering of $\pi^{+}$-mesons by protons at centre-of-mass angles of $43^{\circ}$ and $90^{\circ}$. The meson energy was $43 \mathrm{MeV}$. and a liquid hydrogen target was used, with ring-shaped crystals to define and detect the scattered mesons. By combining the two measurements with an earlier and less accurate determination at $180^{\circ}$, the following values for the $s$ - and $p$-wave phase-shifts in the isotopic spin state $T=3 / 2$ for a meson energy of $43 \mathrm{MeV}$. were obtained : $\alpha_{3}=-4.3^{\circ} ; \quad \alpha_{33}=+4.4^{\circ} ; \quad \alpha_{31}=-1 \cdot 35^{\circ} . \quad$ A. Roberts (Rochester, U.S.A.) in a later paper pointed out that in a scattering experiment the effects of $\pi$-mesons in more than one state of orbital motion with respect to the scattering centre are usually superimposed. In a $\pi^{-}$-mesonic atom, however, in which a $\pi^{-}$-meson exists for a finite time in a Bohr orbit about the central nucleus, pure states of orbital motion exist. The electromagnetic transitions in such an atom thus directly relate the energies of states such as $1 s, 2 p, 2 s, 3 p$. The Coulomb energies of these states can be calculated very accurately, and the nuclear shift of the energy-levels can then be deduced from experimental observation of the mesonic X-rays. Dr. Roberts described an accurate determination of the $2 p-1 s$ transition energy $\left(K_{a}\right.$ and $\left.K_{\beta}\right)$ in the mesonic $K$-series of oxygen; $40-\mathrm{MeV}, \pi^{-}$-mesons were brought to rest in oxygen, and the $\mathrm{X}$-rays, of energy $164 \pm 4 \mathrm{keV}$., were detected in a sodium iodide crystal. An upward nuclear shift of the $1 s$ level was established, showing that there is repulsion in the $s$-state of motion between a meson and a nucleus. The nuclear shift has also been determined for other atoms by workers at the Carnegie Institute, Pittsburgh; unfortunately, it has not yet been possible to measure it for hydrogen. Some similar observations of the $2 p-1 s$ transition energy in $\mu$-mesonic atoms were reported by F. D. S. Butement (Harwell), whose work was carried out using $\mu$-mesons produced by the decay of $\pi$-mesons from the Liverpool cyclotron. For copper a value of $1.55 \mathrm{MeV}$. was obtained for the unresolved doublet and for lead $5.84 \mathrm{MeV}$. These values agree with the earlier work of Fitch and Rainwater and lead to the expression $R=1 \cdot 19 \times 10^{-13} A^{1 / 3}$ for the electromagnetic radius of a nucleus of mass number $A$.

J. M. Cassels (Liverpool) spoke on total crosssection measurements with positive and negative $\pi$-mesons from the Liverpool cyclotron. It is hoped soon to exploit the intense external beam of the machine as a source of high-energy $\pi^{+}$-mesons, which are usually difficult to extract from orbital accelerators ; but in this work an internal target and the same channel (with reversed cyclotron fields) were used for both $\pi^{+}$- and $\pi^{-}$-mesons. The meson energy was $85 \mathrm{MeV}$., and total cross-sections were found by the transmission method using a scintillation counter telescope. Observations of the loss cross-section were made with different angles of acceptance for the final crystal, so that the loss due to Coulomb and diffraction scattering (counted as elastic processes) could be distinguished from that due to nuclear absorption (counted as inelastic processes). The results presented were :

\begin{tabular}{|c|c|c|c|}
\hline Nucleus & \multicolumn{2}{|c|}{$\begin{array}{l}\text { Absorption cross-section } \\
\pi^{-}\end{array}$} & $\begin{array}{c}\text { Error } \\
\text { (per cent) }\end{array}$ \\
\hline $\begin{array}{l}\text { Carbon } \\
\text { Aluminium } \\
\text { Copper } \\
\text { Cadmium } \\
\text { Lead }\end{array}$ & $\begin{array}{r}367 \\
684 \\
1,420 \\
2,190 \\
3,400\end{array}$ & $\begin{array}{r}283 \\
563 \\
960 \\
1,440 \\
2,280\end{array}$ & 8 \\
\hline
\end{tabular}

The difference between the $\pi^{+}$- and $\pi^{-}$-absorption cross-sections for the heavier nuclei may be explained by a simple Coulomb effect, but this is not adequate to interpret the results for carbon. The nuclear radius for lead deduced from the observed cross. sections is $95 \times 10^{-13} \mathrm{~cm}$., compared with a value of $7 \cdot 2 \times 10^{-13} \mathrm{~cm}$. estimated otherwise, so that the lead 
nuclous appears completely black to both types of charged $\pi$-meson at an energy of $85 \mathrm{MeV}$.

Some details of the interaction of high-energy protons with complex nuclei were presented by workers from Birmingham. W. E. Burcham and J. L. Symonds described a measurement of the absolute cross-section for the ${ }^{12} \mathrm{C}(p, p n)^{11} \mathrm{C}$ reaction with proton energies between 250 and $950 \mathrm{MeV}$. The cross-section for carbon-11 production falls off slowly over this energy-range to a value of $20 \cdot 5 \pm 1 \cdot 1$ millibarns at $950 \mathrm{MeV}$. The ratio of sodium-24 and fluorine-18 activities from aluminium bombarded by high-energy protons to carbon-11 activity from polythene bombarded by the same protons was also found for the same range of energies using the internal beam of the Birmingham synchrotron. D. L. Anderson described a special type of photographic emulsion camera which has been installed in the vacuum system of the Birmingham machine. The magnetic field of the machine was used for resolution, and the energy distribution of protons scattered inelastically from carbon was obtained. For low proton energies the results are consistent with a Gaussian distribution of internal momentum in the carbon nucleus.

The day's proceedings included two technical papers of particular interest. K. J. Le Couteur (Liverpool) described the calculations which he had made on the Tuck-Teng system of beam extraction. These calculations were the basis of the design of the peeler and regenerator fields installed in the Liverpool cyclotron. When tried, it was found that the system behaved as anticipated, and that it was possible to shift the circulating beam laterally a large distance in one turn. About one-fifth of the circulating beam was made to enter a magnetic channel and 3 per cent of the internal beam, with an energy of $380 \pm$ $2 \mathrm{MeV}$., was obtained at the end of the channel. After concentration with a focusing magnet, 2 per cent of the internal beam was measured on a target of about $1 \mathrm{in}$. diameter at a distance of $40 \mathrm{ft}$. from the cyclotron. The resulting flux of $4 \times 10^{10}$ protons per cm. ${ }^{2}$ per sec. is about a thousand times better than has been obtained on other machines, and has been a major contribution to the efficient utilization of existing high-energy accelerators. The accelerators of the future must, however, reach much higher energies than any at present attained, and the development of new ideas in this subject was reviewed by W. Walkinshaw (Harwell). The application of the principle of alternating gradient focusing to synchrotron-type accelerators has been thoroughly investigated and underlies the design of the $25-\mathrm{GeV}$. machines now under construction at Geneva and at Brookhaven. The great expense of these machines has, however, stimulated several groups to consider alternative designs, and those proposed by the Midwestern Universities Research Association of the United States (M.U.R.A. group) were discussed in some detail. These envisage a magnet which is excited by direct current and are described as $F F A G$ (fixed-field alternating-gradient) machines. Essentially, fields of special shape are used to confine a large range of momentum within a small range of radius. Typical design figures were presented. There is also the possibility of a fixed-field alternating. gradient constant-frequency cyclotron for an energy of perhaps $2 \mathrm{GeV}$. with a current of perhaps 100 micro. amperes. The remarkable possibilities for experiment opened up by such designs make it essential to complete detailed studies of their practicability and cost as early as possible.

W. E. BURCHAM

\section{FOOD MICROBIOLOGY AND PUBLIC HEALTH}

A

ONE-AND-A-HALF day symposium on "Food Microbiology and Public Health" formed part of the summer conference organized by the Society for Applied Bacteriology, held in Cambridge during July 3-6. Sixteen papers were contributed by authorities in various aspects of the subject from different parts of the world, and the chairmen at the three sessions were the president of the Society, Mr. S. B. Thomas (Aberystwyth), Colonel H. J. Bensted (Colindale) and Dr. G. S. Wilson (London). Several of the contributors described individual outbreaks of bacterial food poisoning and traced so far as possible the likely sources of the trouble. The whole tone of the meeting was directed not to the dangers of food poisoning in itself, but to the dangers arising from ineffective processing, careless handling or unsatisfactory storage conditions.

The first section of the symposium dealt with milk and synthetic cream products, and $\mathrm{K}$. W. Newell (Colindale) described an outbreak of infection in several families arising from eating chocolate éclairs. Frcal coli and a few Salmonella paratyphi were found in the cake mixture and also in the mixing machine; but the principal contamination was Salm. thompson, traced to a single delivery of an unusual pack of Chinese frozen eggs, from which two other strains were also isolated, pullorum and aberdeen. Describing the method of sampling and of isolating the contaminants, Betty Hoblos and Muriel Smith (Colindale) considered that incubation in selenite medium followed by plating on Leifsen medium containing 1 per cent of sucrose is the most satisfactory method. In reply to questions, Dr. Hobbs said that these organisms can survive for more than a year in the frozen state, and that Salm. pullorum cannot be used as an indicator organism, partly because no really satisfactory isolation media have yet been devised.

Custard was the next topic discussed, and R. M. Fry and Joan Boissard (Cambridge) described a sudden outbreak of sore throat, pyrexia and collapse in one section of an army camp following an evening meal which included this comestible. Most of the cases were reported within $48 \mathrm{hr}$. of taking the meal, and nearly all of the throat swabs yielded cultures of Streptococcus pyogenes, Type 9. The incidence of infection pointed to one cauldron of custard handled by one particular cook as the source of the trouble. Doubts were expressed at first concerning this finding, because the material had been handled hot; but it was shown in the laboratory that, although the bulk of material might be at a high temperature, the skin of the custard was relatively cool and so would allow survival of infected droplets falling on the surface.

Emphasis was placed on the importance of the correct handling of dried milk by H. B. Hawley and Margaret Benjamin (Yeovil), because growth of bacteria, and especially of staphylococci, can take place readily at certain stages of processing. They described the design of a suitable drying plant, and said that growth takes place particularly at the 'evaporated milk' stage. For this reason, regular daily cleaning of the plant is essential. The staphylococcal species in the dried milk are different from those in the original milk, pointing to human or airborne contamination gaining access during processing. Coliforms die out quickly in the dried 